\title{
"Borders" in the Writing of Academic Texts: Investigating Informativeness in Academic Journal Abstracts
}

\author{
Gabriela CUSEN \\ Transilvania University of Braşov (Romania) \\ Department of Theoretical and Applied Linguistics \\ gcusen@gmail.com
}

\begin{abstract}
When researchers write academic journal abstracts, they need to meet the requirements of the publisher, which may very well mean that they need to be aware of "the meaning and functions of borders" within which their work is presented in this type of academic text. This paper reports on an investigation of the use of vague language (VL) and IMRaD moves (Introduction, Method, Results, and Discussion) showing the degree of informativeness of academic journal abstracts published in the "Bulletin of Transilvania University" of Braşov between 2010 and 2017. The areas of research these articles focus on range from linguistics and literature to business studies, medicine, and engineering. The analysis of the data, based on Cutting's (2012) analytical framework, revealed that abstract authors use vague language (e.g.: "universal general nouns" and "research general nouns") and that their abstracts mostly consist of introduction, method, and discussion moves. Results of similar research into the writing of article abstracts may be informative for both novice academic text writers and expert writers guiding their work.
\end{abstract}

Keywords: vague language, IMRaD moves, degree of informativeness, universal general nouns, research general nouns.

\section{Introduction}

Academic discourse appears to be a common concern for research in many areas of English studies. In most of the more modern studies in this field, researchers focus on the social function of academic writing since such texts seem to involve interactions between writers and readers (Hyland 2004). Writers and readers of academic texts are members of scientific communities of practice, and the language they have in common may sometimes have specific characteristics. In 
this respect, in the recent years, there has been a growing interest among language researchers in vague language (VL) in academic written discourse. Despite the relatively large number of studies examining the use of vague language in English written academic discourse, there are not very many studies investigating this issue in relation to scientific paper abstracts and even less focusing on the Romanian context. This study, therefore, is an attempt to bridge such a gap.

Before dealing with some definitions of vague language which I am drawing on in the analysis of the data I will present further on, I need to say that "[v]agueness is present in a great deal of language use, and (...) therefore a complete theory of language must have vagueness as an integral component" (Channell 1994: 5). In the same line of thought, the author adds that in the literature "[t]here appear to have been two contradictory points of view: one that vagueness in language is a bad thing, the other, that it is a good thing”. My discussion in this paper starts from the premise that vague language is a good thing, and it is very much worth examining.

\section{Vague language (VL): Definitions}

This section is an attempt at defining the concept of vague language. This is purposefully "an attempt" because there seems to be "more than one perception and definition of vague language (VL), and [...] it can have an informal, socially cohesive function" (Cutting 2007: 3). Thus, according to Channell (1994: 193), since "[a]ny social group sharing interests and knowledge employs nonspecificity in talking about their shared interest", this "non-specificity", i.e. VL, can be understood as a central characteristic of the use of both written and spoken language in daily life. Channell (1994: 20) also affirms that an expression or word is vague if "(a) it can be contrasted with another word or expression which appears to render the same proposition; (b) it is purposely and unabashedly vague or (c) the meaning arises from intrinsic uncertainty".

Lack of precision and fuzziness are characteristics of VL that Cutting (2012: 284) also focuses on in her definition. In this author's words, “... vague language (VL) [is] defined as forms that are intentionally fuzzy, general, and imprecise ... and are heavily dependent on shared contextual knowledge for their meaning". The other important feature of VL, which is clear in her view, is its dependence on both the writer's/speaker's and reader's/listener's common knowledge of the context.

In a similar line of thought, Trappes-Lomax (2007: 122) contends that users and receivers of VL do so on purpose in order to give their understanding of what actually happens in the process of communication, the degree of preciseness that is necessary for the interaction to be meaningful without unnecessary detail. $\mathrm{He}$, therefore, states that "an instance of VL [is] any purposive choice of language designed to make the degree of accuracy, preciseness, certainty or clarity with 
which a referent or situation (event, state, process) is described less than it might have been" (my emphasis).

\section{Vague language (VL): Forms and functions}

While the definition of VL seems to be a matter of fine-tuning among specialists in the field, so do their efforts to agree on a taxonomy of the various types of VL. This may be because, in Drave's (2001: 26) terms, they "share the property of propositional imprecision".

Any discussion of VL would not be complete if the name of Channell (1983, 1985, 1990, 1994) and her work in the domain were not mentioned. Her work on the use of VL in both spoken and written language has been inspirational for many other researchers. Drave (2001: 26), for example, adopts Channell's analytical framework in his investigation of intercultural conversations. He describes the following types of VL: number approximations such as "about 12, 5 or 6". Here he mentions a "sub-category of 'partial specifiers' [that] indicates that the approximation is either more or less than the named figure, e.g. at least 20 , under 100". The next category is that of non-numerical vague quantifiers, and the author states that here "[v]agueness is indicated by vague words or phrases rather than by using numbers e.g. several people, a lot of books". Vague category markers represent another category of VL, and they consist of vague "tag[s]" added to lexical items in order to show that such items are examples of the category itself. Drave exemplifies with "fish or something, lectures and so on". The final category this author defines is that of "placeholder words", whose role is to replace words with a more specific meaning. His examples are "thing, stuff".

Cutting (2012: 284) also reviews various works on VL and claims that it seems that researchers' interest in the area of VL in written academic English resides mainly in vague quantifiers and hedging devices; general nouns and general extenders ("list completers") have also been the focus of some research, whereas general verbs and indefinite pronouns appear to have attracted less attention.

In this paper, I adopt Cutting's (2012) analytical framework, the investigation of the types of VL, and their functions in analysis of the data in the corpus this paper reports on. This author deals with several categories of VL in her work on conference abstracts. Here, I only give details on those types of VL described in the literature (see, for example: Kennedy 1987, Channell 1994, Ruzaite 2004, Cutting 2012).

Thus, vague quantifiers are seen as being used as a matter of convenience when language users feel that more precision is not necessary. Cutting's (2012: 285) examples are "'about 30 subjects', 'around 18 million', 'a great deal of time', 'a number of samples'”. The category of hedging devices is represented by language 
items which are primarily used by language users to show their "commitment to the truth value of their proposition". To exemplify, the author states that "[s]uch hedges are: catenatives (verbs taking non-finite complements, e.g.: appear to, suggest that), modal operators (might), and modal nouns (probability), adjectives (likely) or adverbs (possibly)". These types of VL were also the focus of research by Hyland (1996) and Salager-Meyer (1994).

Finally, I will give some more attention to the category of general nouns which was best represented in the data analysed in this study. Halliday-KirkwoodHasan (1976) consider that they are "dummy" nouns such as thing, people, and place. For Mahlberg (2005), general nouns are high-frequency nouns whose meaning is related to the functions of texts or to co-text. In Cutting's work (2007: 224), [t]he general nouns ... are ones that are not lexically cohesive and they refer to a specific entity known by speaker and hearer", or writer and reader in the case of written academic writing. Moreover, she considers that this category of vague nouns can have two sub-divisions: "universal general nouns as: feature(s), factor(s), issue(s)" and "research general nouns" such as: "aim, research, analysis, method" (2012). The author also states that in her research on conference abstracts both universal general nouns and research general nouns are either pre- or post-modified or both, which does not necessarily make their meaning less vague. Here are some examples she gives to this effect: "significantly better performance" or "aspects of meaning" (2012, emphasis in the original).

A review of the literature of VL suggests that there are two major functions of various forms of VL, i.e. to provide textual cohesion and to show membership of a community of practice. Firstly, Cutting (2012: 285) shows that the provision of textual cohesion is mainly a function of general nouns "which gain meaning from cohesion". A major function of general words is to provide textual cohesion. Similarly, Aktas-Cortes (2008) agree that general nouns gain meaning from cohesion and call them 'shell nouns' since they both enclose and anticipate the surrounding text. Flowerdew (2003) names these nouns 'signalling nouns' and explains that they signal a relationship with the surrounding text. Ivanic (1991: 109) also deals with VL and explains that such language is "imbued with both a constant dictionary meaning and a variable specific meaning, dependent on the context". She observes that "on occasion a meaning cannot be recovered from the surrounding discourse". The study I am reporting on here focuses on those general nouns which gain their meaning from textual cohesion.

Secondly, the other major function of VL is to show membership of a community of practice, i.e. it has an interpersonal function. This is discussed below from the perspective of Cutting's (2012: 285) work on conference abstracts and in relation to what she calls: "hedging devices whose functions may be: courtesy, modesty, caution" and "non-hedging devices whose functions may be: convenience, selfdefence, power, and anticipation" (emphasis in the original). Briefly, according to 
this author, the hedging function of "courtesy" is present in writing, which shows that writers are "certain of their assertions but choose to use either positive or negative politeness strategies out of respect for the assumed prior knowledge of the audience". The function of "modesty" characterizes the work of those authors who "are certain of their assertions" but prefer "to hedge by using an imprecise reference to display humility in order to minimise the threat to their own face". The third hedging function that Cutting (2012: 285) details is "caution". In this situation, the researcher refers to her work on conference abstracts and claims that writers' use of VL "can express tentativeness about inconclusive data, which may be the case when hedging is the strongest claim a careful researcher can make - VL is used to honestly express scientific scepticism and doubt". In the larger category of the non-hedging functions of VL, the first one described by Cutting is that of "convenience", which shows that authors may use VL "possibly because they feel that further precision would not contribute substantially to their argument". Next, "self-defence" is a function which points to the author's move to avoid precision in order to "minimise the threat to their own face".

One very interesting function of VL is that of exerting "power", which is a function that is quite the opposite of hedging. In this area, the seminal work of Fairclough (1989: 82-83) must be mentioned. This author states that "texts that exert an influence over the reader impose implicit assumptions upon the audience by encouraging them to bring the same assumptions to the process of interpretation". And finally on the functions of VL in academic writing: the last of the non-hedging functions described by Cutting (2012: 285) - "anticipation" - appears to fit well with the "promissory nature" of academic journal abstracts I deal with in this paper. This is so because authors may use VL because they "prefer not to talk in detail about their results [and] they do not want to give away the denouement of their study", and in this way they wish to rouse the curiosity of the reader leafing through an academic journal.

\section{IMRaD structure of academic journal article abstracts}

If the pervious sections have dealt with the first major perspective from which I investigate academic journal abstracts, i.e. the use of VL, in this section, I focus on the structure of these texts based on what representatives of the literature in the field (Kaplan et al. 1994, Swales-Feak 2009, Cutting 2012) discuss as IMRaD structure. IMRaD, therefore, is considered to be a norm for the structure of an academic text such as a journal abstract. It focuses on the organization of the content of the text and stipulates that there are sections, or "moves", in such texts which authors need to abide by in order for their abstracts to meet the required "degree of informativeness in terms of the referential explicitness of 
academic text" (Cutting 2012). In other words, IMRaD structure is meant to set certain "borders" within which academic texts are placed. Moreover, since the abstract supposed to function as an autonomous text, the "moves" it consists of are: the Introduction, in which a reader should find the context of the study the author reports on, its aims, and a very brief literature review which places the work in the area it belongs to. In the Method move, the author "explains how the data was collected and analysed". The Results move "provides summaries of findings". Finally, the Discussion move "offers interpretations, implications, and applications" (2012: 284).

\section{Data in this study and corpus}

The data corpus investigated in this study consists of a number of 2,349 academic journal article abstracts. The source of these data was the electronic version of the Bulletin of Transilvania University of Braşov, Romania, Series I to IX, Nos 1 and 2 published between 2010 and 2017. The language of this version of scientific publication is English. The areas of research the Bulletin focuses on and the number of abstracts collected from each series are: Series I - Engineering sciences (272 abstracts), Series II - Forestry. Wood industry. Agricultural food engineering (229 abstracts), Series III - Mathematics. Informatics. Physics (230 abstracts), Series IV - Philology and cultural studies (241 abstracts), Series V Economic sciences (467 abstracts), Series VI - Medical sciences (202 abstracts), Series VII - Social sciences and law (350 abstracts), Series VIII - Performing arts (174 abstracts), and Series IX - Sciences of human kinetics (184 abstracts).

These data are analysed in what follows from the two perspectives detailed in the previous sections of this paper: a) use of vague language and b) degree of informativeness in terms of IMRaD moves.

\section{Data analysis}

In what follows, I discuss the forms and functions of VL identified in the data and the IMRaD organization of the academic journal abstracts this study investigates. The paper addresses the following questions:

1. What are the forms and functions of VL identified in the corpus?

2. What is the distribution of IMRaD moves in the abstract corpus?

3. How are IMRaD moves and forms and functions of VL related?

Besides setting the boundaries of this study, the first two questions are aimed at guiding the analysis of the data in terms of both the identification and discussion of forms and functions of VL and the examination of the abstracts in the corpus 
from the perspective of the distribution of the moves that may or may not give them the required degrees of informativeness such a text is supposed to offer to interested readers. Question three, on the other hand, directs attention towards the presence or absence of VL in the four moves these abstracts may consist of.

\subsection{Vague language (VL) forms and functions: This study}

In this section, the analysis focuses on forms and functions of VL, and the examples discussed here come from article abstracts published in some of the nine series of the Bulletin of Transilvania University of Braşov. In the examples below, vague structures are bolded, and at the end of each abstract extract, in between round brackets, the name of the bulletin series is mentioned.

That both speakers and writers use vague language is common knowledge. In Cutting's words (2007: 6), this is the use of "intentional vagueness, which occurs by choice". It is the abstract authors' "choice" to use VL that is exemplified and discussed below. However, from the beginning of this discussion, mention needs be made that when it comes to the types of VL identified in this study, while the categories of vague quantifiers and hedging devices are not very much noticeable, those of universal general nouns and research general nouns pervade most of the abstracts in the corpus.

The examples below are of abstract authors' use of vague quantifiers:

(1) Which choice is to be considered adequate is to be discussed on the basis of several theoretical points of view appropriate to the field of translation criticism. (Philology and Cultural Studies: Language and Linguistics)

(2) Histological study of muscle and bone in the same patient shows a clear lesion parallelism, proving that some of the blame for the production of the fracture falls upon the muscle and bone. (Medical Sciences)

(3) Satellite images are taken with a wide variety of sensors placed on platforms at altitudes between 400 and $950 \mathrm{~km}$. They are characterized by a number of elements specific to each satellite program amongst which the most important are resolution (spatial, spectral, radiometric, temporal), setting size, applications where records can be used, the image cost. (Forestry)

As already stated, in this corpus, very few such instances of use of vague quantifiers ("several", "some", and "a number of" in the examples above) were identified, and their functions appear to be either "convenience" or "caution" (Cutting 2012). Convenience then, a non-hedging function, indicates that authors 
may show that further precision would not contribute to their arguments. Caution, on the other hand, is a hedging function showing that the author expresses tentativeness about their research and honestly expresses scepticism and doubt.

An interesting case here is the use of "at least" in example (4) below. This is a "partial specifier" (Channell 1994) indicating that authors use it to state that the named figure is either more or less than it actually shows. The functions of such a quantifier may again be caution.

(4) The Ahlfors Q-regular spaces are a natural setting for the theory of quasiconformal mappings since in these spaces the three definitions of quasiconformality in Euclidean spaces of dimension at least two, can be formulated, but they are not equivalent.

Examples (5) and (6) illustrate authors' use of hedging devices:

(5) Also, empirical approaches in the skylines design process may lead to poor technical solutions with repercussions both on work safety as well as on the installation maintenance. (Forestry)

(6) What could we possibly learn from a 70 year correspondence from the years of the Second World War, when the course of subsequent history has already been known? The sociologist is interested in seeing how an event unfolds, how it generates other actions, how the actors are driven by, what their strategies are and how they interact. (Social Sciences)

In the two examples above, the hedging devices ("may" - a modal operator and "possibly" - an adverb) are used to qualify the authors" commitment to the truth value of their claims. Their functions may be either modesty in that the authors prefer to hedge in order to show humility and to minimize the threat to their own face or caution, which indicates that the authors show they are responsible and honest researchers and express some scientific doubt in relation to the quality of technical solutions in Example (4) or the implications of sociological research in Example (5).

Next, in the examination of VL forms and their functions, this section deals with the two categories of general nouns detailed by Cutting (2012), i.e. universal general nouns and research general nouns. These two types of vague language are best represented throughout the journal article abstract corpus under examination in this paper. The universal general nouns most often used in this corpus in either their singular or plural forms were: feature(s), factors, context, practices, implications, actions, activities, feature(s), situation(s), resource(s), aspect(s), and effect(s). On the other hand, the research general nouns most favoured by 
the authors were: aim, study, research, data, analysis, finding(s), result(s), project, investigation, method, methodology, objective, procedure, and conclusion. A large proportion of both universal general nouns and research general nouns identified in the corpus have a variety of either pre-head modifiers or post-head modifiers or both, which seem to serve nouns to gain little meaning or to limit the categories of meaning, and thus they remain relatively vague. Both categories of general nouns examined here collocate with such verbs as demonstrate, indicate, obtain, include, or show. Below, there are two examples of the use of universal general nouns.

(7) Retirement, the dissolution of the social network, the essential rearrangement of the "marriage nest" are the causing factors of the change or loss of identity during the third age, which can lead to the perception of senior citizens' lives in a way that is totally different from what is considered to be normal from a social point of view. (Social Sciences)

(8) The most important usability aspects of integrating such a sensing dimension to modern vacuum cleaners have been explored in this paper, along with a trivial implementation, to assure the portability and compactness of the cleaning appliances. (Engineering Sciences)

In Example (7) (and in most of the corpus), the pre-head modifier simply limits the category of meaning, which gives the noun a relatively vague sense. Readers have to conclude from the co-text what these factors are. Here the function may be convenience or anticipation. In (8), there is an example of general noun having a post-head modifier, which seems to equate to partitives with a support function. Importantly, the authors of all abstracts in this corpus used general nouns in similar ways.

A clearly distinguishing feature of the academic journal abstracts examined in this study is their quite high density of what Cutting (2012) calls "research general nouns". The research general nouns most frequently present in the data were exemplified above. Examples (9) and (10) below contain research general nouns whose functions may be convenience (in 9) because the author addresses a reader who is presumably knowledgeable in the area of research methodology and anticipation (in 10) since the pre-head modifier additional does not make the noun less vague and the structure itself goes in line with the "promissory nature" (Cutting 2012: 286) of the abstract.

(9) The results of the analysis show that, although some protectionist tendencies have been recorded both in extra[-] and intra-EU trade, trade relations which are provided among member states are of significant importance all the time. (Economic Sciences) 
(10) Additional research is needed to disclose the interaction between the dysfunction of the lower urinary tract and practicing of sport. (Sciences of Human Kinetics)

After having dealt with ways in which VL is used by writers of academic journal abstracts, I next discuss the structure of the abstracts in the corpus in terms of the distribution of the IMRaD moves in these texts.

\subsection{IMRaD moves in academic journal article abstracts: This study}

That scientific article abstracts must be well structured in order to be informative and therefore helpful for interested readers is a common point made by specialists in the domain. Studies dealing with academic paper abstracts (Kaplan et al. 1994, Swales-Feak 2009, Cutting 2012) have shown that in order for such texts to meet the required degree of informativeness they have to abide by a certain distribution of IMRaD moves (Introduction, Method, Results, and Discussion). In some more detail and in relation to the examples below, the Introduction move includes information about context, aims, and literature review (coded here as: [Intr.: context, aims, lit rev.]), the Method move gives details about data collection and analysis (coded here as [Meth.]), the Results move focuses on findings (coded here as [Res.]), and the Discussion move contains explanations, implications, and applications (coded here as [Disc.]).

Before the discussion of IMRaD moves in the abstracts examined in this study, in Example (11) below, there are instructions to authors who decide to publish their work in the Bulletin of Transilvania University.

(11) Abstract: These instructions are meant to help with the template for the articles published in the scientific journal Bulletin of Transilvania University of Braşov. The material presents the camera-ready template for the articles. The abstract should synthetically outline all the pertinent results in a short but intelligible form. The abstract should begin by clearly stating the purpose of the paper and should end by formulating the most important conclusions. Short, direct and complete sentences will be used and they will be written in a single paragraph, without "tab"-s. The abstract will be 7-10 lines long.

One can easily see that these instructions seem to focus on the introduction, the results, and the discussion sections of the articles and disregard the methodology move. Moving on to the abstracts in the corpus examined here, as anticipated, their IMRaD structure did not have an equal distribution of moves. 
Table 1 below gives a quantitative view of the distribution of IMRaD moves in the corpus. First, the most interesting point here seems to be that the difference between the number of abstracts displaying all four moves (535) and those that only contain one such move (583) is not that great, which might mean that at the two extremes there are authors who are either well informed in what concerns the writing of abstracts or have other concerns in mind than the writing of these article introductory texts. Second, the distribution of IMRaD abstract moves among the nine series of the publication shows considerable variety, which might be a matter of concern for its editors.

Table 1. Distribution of IMRaD moves in the corpus

\begin{tabular}{ll}
\hline No of moves \& no of abstracts & Bulletin of Transilvania University/ Series \\
\hline All 4 moves $=535$ & IV. Phil. \& CS; V. Ec. SC.; VI. Med. Sc.; \\
& VII. Soc.sc.; IX Hu. Kin. \\
\hline 3 moves (Introduction, Method, & I. En. sc.; II. For.; III. Math.; IV. L\&L; V. Ec. \\
and Discussion) $=742$ & sc.; VII. Soc. sc.; VIII. Perf. arts \\
\hline 2 moves (Introduction and & I. En. sc.; II. For.; III. Math.; IV. Phil. \& CS.; \\
Discussion) $=489$ & V. Ec. sc.; VI. Med. Sc.; VII. Soc. sc.; IX Hu. \\
\hline 1 move (Introduction/ & Kin. \\
\hline Methodology/Discussion) $=583$ & I. En. sc.; II. For.; III. Math.; IV. Phil. \& CS.; \\
& V. Ec. sc.; VI. Med. sc VII. Soc. sc.; VIII. Perf. \\
& arts; IX Hu. Kin. \\
\hline
\end{tabular}

The examples below are full abstracts in which the distribution of moves ranges from all four to only one. Moreover, in these four abstracts, the bolded words are examples of VL use, and in the discussion of each abstract they are related to the move they belong to.

(12) [Intro: context] The present study aims to explore the manner in which women political leaders construct their public and personal identity in and through the media, by identifying the topics discussed and the discursive strategies employed. [Meth.: data collection and analysis] The corpus is formed by 15 media interviews given by and articles about two Italian women politicians, Emma Bonino and Laura Boldrini. The framework applied is the discursive-historical approach (e.g. Reisigl and Wodak 2009). [Res.] The results show that the women speakers attempt to construct their identity in a composite manner, uniting values such as firmness and courage with empathy and sensitiveness to social issues. [Disc.: implications] Such selfpresentations aim to project the speakers as role models and to encourage social change. (Philology and Cultural Studies) 
Example (12) is an abstract that contains all four IMRaD moves. The introduction provides the context of the study, then the method clearly shows what data the study relies on and how they were analysed. The results and discussion moves lead the reader towards an idea of the findings and the implications of such a study. In what VL is concerned, this example is also representative for the majority of the corpus since the author uses mostly research general nouns with a relatively vague meaning. Moreover, these instances of VL use are present in all four moves in the abstract.

(13) [Intr.: aim] The aim of this study was to analyse the relationship between the expression of the bcl-2 proto-oncogene and the histological grading of prostate cancer according to the Gleason score. [Meth.] To study the prognostic significance of Bcl-2 over expression in prostate cancer, 36 consecutive radical prostatectomy specimens were examined by immunohistochemistry. Bcl-2 was associated with malignant phenotype. Bcl-2 over expression (found in $14-38.89 \%$ tumours) was associated with high Gleason score. [Disc.] These data suggest that altered expression of Bcl-2 plays a role in prostate cancer progression. (Medical Sciences)

(14) [Intr.] Up to this day, many cost-effective vision systems have been successfully used in various domains (such as manufacturing, human machine interaction, robotics, automotive and so on) to provide a new sensing dimension of the working environment. However, there are no such systems implemented for modern manual-controlled vacuum cleaners. [Meth.] The most important usability aspects of integrating such a sensing dimension to modern vacuum cleaners have been explored in this paper, along with a trivial implementation, to assure the portability and compactness of the cleaning appliances. (Engineering Sciences)

(15) [Meth.] In this paper we study the geometry of the complex indicatrix of a complex Cartan space. We prove that the intrinsec Chern-Cartan complex nonlinear connection of the indicatrix coincides with the induced Chern-Cartan complex nonlinear connection. Also, the induced Chern-Cartan linear connection on the complexified indicatrix is studied and the existence of an almost hermitian contact structure is proved. The notions are introduced in similar manner with the corresponding results from complex Finsler geometry. (Mathematics)

Other than representing examples of those abstracts in the corpus in which less than four moves were identified, excerpts (13), (14), and (15) also show the use of VL, i.e. universal general nouns, research general nouns, and vague quantifiers. 


\section{Conclusions}

To conclude, I now go back to the questions in section 6 in an attempt to answer them. As the analysis of the data shows, the scientific journal abstracts investigated here mainly consisted of "introduction", "method", and "discussion" (IMRaD) moves. The "results" move seems not to be very popular with the authors of these abstracts. In these moves, the VL forms are mostly universal general nouns (e.g.: feature(s), factors, context, practices, implications) and research general nouns (e.g.: aim, study, research, data, analysis, finding(s), result(s)). Both universal general nouns and research general nouns had pre-head modifiers and less often post-head modifiers that did not add much meaning. In addition to the function of textual cohesion, general nouns fulfilled hedging ("courtesy" and "caution") and non-hedging ("convenience" and "anticipation") functions.

This study is of an exploratory type, wherefore the conclusions of the analysis of the data corpus are not generalizable. However, the fact that the data are drawn from nine areas of academic research may give the study an internal validity. In terms of the applicability of its results, it may be that such research into the writing of article abstracts will benefit both novice academic text writers and academics guiding their work. Finally, further research dealing with data (abstracts) from similar research areas published in other national or international scientific journals could explore how their IMRaD moves and use of VL differ from those investigated in this study, and thus a certain degree of external validity could be reached. Moreover, coding and analysis of data by a second researcher could give reliability to the results.

\section{References}

Aktas, Rahime Nur-Cortes, Viviana. 2008. Shell nouns as cohesive devices in published and ESL student writing. Journal of English for Academic Purposes 7(1): 3-14.

Channell, Joanna. 1994. Vague Language. Oxford: Oxford University Press.

Cutting, Joan (ed.). 2007. Vague Language Explored. New York: Palgrave Macmillan.

2012. Vague language in conference abstracts. Journal of English for Academic Purposes 11: 283-293.

Drave, Neil. 2001. Vaguely speaking: A corpus approach to vague language in intercultural conversations. Language and Computers 36(1): 25-40.

Fairclough, Norman. 1989. Language and Power. London: Longman.

Flowerdew, John. 2003. Signalling nouns in discourse. English for Specific Purposes 22: 329-346. 
Halliday, Michael-Alexander Kirkwood-Ruqaiya Hasan. 1976. Cohesion in English. London: Longman.

Hyland, Ken. 1996. Talking to the academy: Forms of hedging in science research articles. Written Communication 13(2): 251-281.

Hyland, Ken. 2004. Metadiscourse in academic writing: A reappraisal. Applied Linguistics 25(2): 156-177.

Ivanic, Roz. 1991. Nouns in search of a context: A study of nouns with both openand closed-system characteristics. International Review of Applied Linguistics in Language Teaching 29(2): 93-115.

Kaplan, Robert-Selena Cantor-Cynthia Hagstrom-Lia D. Kamhi-Stein-Yumiko Shiotani-Cheryl Boyd Zimmerman. 1994. On abstract writing. Text 14(3): 401426.

Kennedy, Graeme D. 1987. Quantification and the use of English: A case study of one aspect of the learner's task. Applied Linguistics 8(3): 264-286.

Mahlberg, Michaela. 2005. English General Nouns: A Corpus Theoretical Approach. Amsterdam: John Benjamins Publishing Company.

Ruzaite, Jurate. 2007. Vague Language in Educational Settings: Quantifiers and Approximators in British and American English. Frankfurt am Main: Peter Lang.

Salager-Meyer, Françoise. 1994. Hedges and textual communicative function in medical English written discourse. English for Specific Purposes 13(2): 149170.

Swales, John. M.-Christine B. Feak. 2009. Abstracts and the Writing of Abstracts. Michigan: University of Michigan Press.

Trappes-Lomax, Hugh. 2007. Vague language as a means of self-protective avoidance: Tension management in conference talks. In Cutting, Joan (ed.), Vague Language Explored, 117-137. New York: Palgrave Macmillan. 